\title{
The size of AB Doradus A from VLTI/AMBER interferometry ${ }^{\star}$
}

\author{
J. C. Guirado ${ }^{1}$, J. M. Marcaide ${ }^{1}$, I. Martí-Vidal ${ }^{2}$, J.-B. Le Bouquin ${ }^{3}$, L. M. Close ${ }^{4}$, W. D. Cotton ${ }^{5}$, and J. Montalbán ${ }^{6}$ \\ 1 Dpto. Astronomía y Astrofísica, Universidad de Valencia, Dr. Moliner 50, 46100 Burjassot, Valencia, Spain \\ e-mail: guirado@uv.es \\ 2 Max-Planck-Institut für Radioastronomie, Auf dem Hügel 69, 53121 Bonn, Germany \\ 3 Laboratoire d'Astrophysique de Grenoble, CNRS-UJF UMR 5571, 414 rue de la Piscine, 38400 Saint Martin d'Hères, France \\ 4 Steward Observatory, University of Arizona, Tucson, Arizona 85721, USA \\ 5 National Radio Astronomy Observatory, 520 Edgemont Road, Charlottesville, VA 22903-2475, USA \\ ${ }^{6}$ Institut d'Astrophysique et de Gèophysique de l'Université de Liège, Allée du 6 Août 17, 4000 Liège, Belgium
}

Received 7 June 2011 / Accepted 4 August 2011

\section{ABSTRACT}

\begin{abstract}
The pre-main sequence (PMS) star AB Dor A is the main component of the quadruple system AB Doradus. The precise determination of the mass and photometry of the close companion to $\mathrm{AB}$ Dor $\mathrm{A}, \mathrm{AB}$ Dor $\mathrm{C}$, has provided an important benchmark for calibration of theoretical evolutionary models of low-mass stars. The limiting factor to the precision of this calibration is the age of the system, as both the mass and luminosity of $\mathrm{AB}$ Dor $\mathrm{A}$ and $\mathrm{C}$ are well monitored by other ongoing programs. In this paper we present VLTI/AMBER observations of AB Dor A which provide a direct measurement of the size of this star, $0.96 \pm 0.06 R_{\odot}$. The latter estimate, combined with other fundamental parameters also measured for this star, allows a precise test of PMS evolutionary models using both H-R diagrams and mass-radius relationships. We have found that our radius measurement is larger than that predicted by the models, which we interpret as an evidence of the oversizing produced by the strong magnetic activity of AB Dor A. Considering, at least partially, this magnetic effect, theoretical isochrones have been used to derive constraints to the age of $\mathrm{AB}$ Dor $\mathrm{A}$, favouring an age about 40-50 Myr for this system. Older ages are not completely excluded by our data.
\end{abstract}

Key words. stars: pre-main sequence - stars: fundamental parameters - techniques: interferometric - stars: individual: AB Doradus

\section{Introduction}

$\mathrm{AB}$ Doradus is a quadruple stellar system, consisting of two close pairs, AB Dor A / AB Dor C and AB Dor Ba / AB Dor Bb, separated by about $9^{\prime \prime}$. The brightest star of the system, $\mathrm{AB}$ Dor A, is a well-known, pre-main sequence (PMS) K1-star, with strong emission at all wavelengths, from radio to X-rays. Among other instruments, AB Doradus has been observed by the HipPaRcos satellite, very-long-baseline-interferometry (VLBI) arrays (Guirado et al. 1997) and different near-infrared instruments at the VLT (Close et al. 2005; Close et al. 2007; Boccaletti et al. 2008). One of the main results from these observations is the independent measurement of both the JHK photometry and the dynamical mass of AB Dor C $\left(0.090 \pm 0.005 M_{\odot}\right)$, the companion to $\mathrm{AB}$ Dor $\mathrm{A}$. Hence, $\mathrm{AB}$ Dor $\mathrm{C}$ is a unique object to calibrate theoretical mass-luminosity relations; actually, this calibration has shown that theoretical evolutionary tracks tend to underestimate the mass of very low mass objects (Close et al. 2005).

Ongoing observing projects on AB Doradus are dedicated to monitoring both the reflex orbit of AB Dor A (via VLBI techniques with Australian antennas) and the differential orbit between AB Dor A and AB Dor C (via near-infrared VLT observations). As the above-mentioned observations will improve the photometry and the dynamical mass of $\mathrm{AB}$ Dor $\mathrm{C}$, the successful calibration of evolutionary models with $\mathrm{AB}$ Dor $\mathrm{C}$ measurements will be limited by the uncertainty in the estimate of the age of $\mathrm{AB}$ Dor $\mathrm{A} / \mathrm{AB}$ Dor $\mathrm{C}$. This parameter is still a matter of

^ Based on observations made with ESO Telescopes at the Paranal Observatory under programme ID 384.C-1053. discussion, with different estimates in the literature: Zuckerman et al. (2004) first proposed an age of 50 Myr (later supported by López-Santiago et al. 2006); on the other hand, Luhman et al. (2005) and Ortega et al. (2007) estimate an older age for this system, $120 \pm 20 \mathrm{Myr}$; intermediate ages, $75 \pm 25 \mathrm{Myr}$, have been reported by Nielsen et al. (2005), Janson et al. (2007) and Bocaletti et al. (2008). This relatively wide range of ages remains as the largest ambiguity to test model predictions with AB Dor C. In this scenario, the measurement of fundamental parameters of any of the members of the stellar system may be used to constrain evolutionary models and/or derive bounds to the age of the system. In particular, as PMS stars change in radius as they contract to the main sequence, having a precise determination of the size of AB Dor A will serve, accordingly, to constrain the age of the system.

In this paper we present a precise determination of the size of AB Dor A from VLTI observations performed with the AMBER focal instrument (Petrov et al. 2007), installed at the ESO facilities in Cerro Paranal, Chile. In Sect. 2 we describe the observations and data reduction; in Sects. 3 and 4 we report the results and discussion, respectively. Conclusions are presented in Sect. 5.

\section{Observations and data reduction}

We observed AB Dor A with the VLTI using the AMBER instrument at low spectral resolution mode in the $J, H$, and $K$ bands. The observations were performed on 26 December 2009, from 00:30 to 10:30 LST, using the 2 m-class Auxiliary Telescopes (ATs) placed on stations A0, K0, G1; for cycle 84A, these 
Table 1. Parameters of the star calibrators used in our observations.

\begin{tabular}{cccc}
\hline \hline & $\begin{array}{c}\text { Angular distance } \\
\text { to AB Dor A }\left({ }^{\circ}\right)\end{array}$ & K magnitude & $\begin{array}{c}\theta_{\mathrm{UD}} \\
\text { (mas) }\end{array}$ \\
\hline HD 35199 & 2.7 & 3.96 & $0.859 \pm 0.012$ \\
HD 39608 & 5.3 & 3.83 & $0.945 \pm 0.012$ \\
HD 39963 & 2.8 & 4.36 & $0.638 \pm 0.009$ \\
\hline
\end{tabular}

Notes. Values for $\theta_{\text {UD }}$ correspond to diameters calculated from a uniform-disk model (Mérand et al. 2005).

stations provided the maximum angular resolution for our observation, 2.3 milliarcsec (mas). Each AMBER observing block contains 5 exposures of the target (AB Dor A) or calibrator (see below) and two additional exposures for dark and flat correction, each exposure having 200 frames, each recorded with a DIT of 50 milliseconds. Target and calibrator were observed alternatively. To ensure a proper amplitude calibration, we used three different calibrators, namely HD 35199, HD 39608, and HD 39963 (see Table 1), each one with size reported in Mérand et al. (2006). We selected HD 35199 as primary calibrator and we scheduled it throughout the complete observing run along with $\mathrm{AB}$ Dor A. Not to excessively compromise the CAL-SCI duty cycle, we included, only after 04:30 LST, and alternatively, observations of HD 39608 and HD 39963. The redundancy provided by multiple calibrators allows a test of the quality of the calibration of the visibility amplitudes.

The raw data frames were transformed to individual complex visibilities following the standard routines of the amdlib libraries (version 2.2; Tatulli et al. 2002) that we outline briefly here. First, using target and calibrators frames, we corrected for the spectral displacement between the photometric spectra of the ATs and the interferometric spectrum; second, we removed the bad pixels and applied the DARK and FLAT corrections; third, we removed the instrumental dispersive effects, i.e. fringe-fitted each frame using the P2VM algorithm in amdlib; and fourth, we selected and averaged the frame visibilities resulting from P2VM to obtain a single visibility for each exposure and spectral channel. We made extensive tests to determine the appropriate selection criteria (based on consistency and robustness of the results; see Sect. 2.1), which were found to be the following: we kept frames with atmospheric piston smaller than $8 \mu \mathrm{m}$ and time between 02:15 and 07:10 (to avoid low-elevation observations), keeping the $50 \%$ of the remaining frames with highest SNR (highest fringe contrast). Finally, we extracted and averaged all the selected exposure visibilities within each observing block using python-based algorithms (Martí-Vidal et al. 2011) to obtain the complex visibilities. From a first inspection of the visibility amplitudes, we edited out those with low-quality, most of them of the $J$-band. Standard deviations of $2-5 \%$ were obtained for the remaining visibility amplitudes.

\subsection{Amplitude visibility and wavelength calibration}

AMBER amplitude is usually calibrated by measuring the transfer function of a star with known size, which is interpolated to the target star times, assuming the transfer function remains unchanged (Tatulli et al. 2002). In our observations, the transfer function was calculated by comparing the measured visibility amplitudes of the main calibrator HD 35199 with the predictions from a uniform-disk (UD) model with the diameter shown in Table 1. The accuracy of the estimate of the size of AB Dor A depends to a high degree on the quality (i.e. spatial and temporal stability) of the transfer function obtained from HD 35199. To ascertain this quality, we used HD 35199 to calibrate the amplitudes of the other two CAL stars, HD 36908 and HD 39963, both with known size as displayed in Table 1. Should the HD 35199 be well edited and calibrated, a modelfit process must provide UD-diameters for the two secondary calibrators similar to those in Table 1. We found optimal coincidence between tabulated and modelfitted diameters for the selection criteria described in Sect. 2.

The lack of spectral calibration for AMBER leads to an uncertainty in the observing wavelength $\lambda$. This effect can be manually corrected by means of the identification of absorption lines present in the data of the target star and/or the calibrators (i.e. Martí-Vidal et al. 2011). However, finding absorption features in LR-mode AMBER observations is difficult; in fact, our data does not show any atmospheric signature pronounced enough to be used for a precise spectral calibration. Hence, we were forced to adopt a different approach, using again the two secondary calibrators in a similar procedure to that described above. We explored the solution (i.e. UD-diameter for both secondary calibrators) using shifts in $\lambda$ between -0.15 and $0.15 \mu \mathrm{m}$; this quantity looks conservative in view of other estimates at low- and medium-resolution AMBER observations (Domiciano de Souza et al. 2008; Kraus et al. 2009; Martí-Vidal et al. 2011). Within this range, we found that a shift of $+0.1 \mu \mathrm{m}$ minimized the differences between our modelfitted sizes and the nominal sizes displayed in Table 1. Final modelfit estimates of the UD-diameters were $0.95 \pm 0.04$ mas for HD 39608 and $0.66 \pm 0.04$ mas for HD 39963, where the standard deviations shown included the contribution of this wavelength calibration process (we conservatively increased the statistical errors, \pm 0.02 mas, to cover the scatter found in our exploratory approach). Once the goodness of the transfer function was assessed, we calibrated the visibility amplitudes of AB Dor A similarly, using HD 35199 data.

\section{Results}

The observed and calibrated visibilities of AB Dor A (see Fig. 1) show that $A B$ Dor A is partially resolved by the A0-K0-G1 interferometer, as expected for a solar-size star placed at the AB Dor A distance. Modelfit of the visibility amplitudes to a uniform-disk model gives a size of AB Dor A of $0.62 \pm 0.04$ mas. We note that the UD model is a crude approximation of the stellar surface brightness distribution, and that other models may provide a more realistic representations, i.e., a limb-darkened disk model (LD model), with decreasing intensity toward the edge of the stellar disk. The estimate of a LD diameter directly from the AB Dor A visibilities looks difficult, given the limited sampling of the first lobe of the visibilities, which is probably not enough to discriminate between different LD sizes. Instead, following Di Folco et al. (2004), we converted the UD-diameter $\left(\theta_{\mathrm{UD}}\right)$ to LD-diameter $\left(\theta_{\mathrm{LD}}\right)$ using the approximate expression given in Hanbury-Brown et al. (1974),

$$
\rho(\lambda)=\frac{\theta_{\mathrm{UD}}}{\theta_{\mathrm{LD}}} \sqrt{\frac{1-u(\lambda) / 3}{1-7 u(\lambda) / 15}}
$$

where the coefficients $u(\lambda)$ for our spectral bands were taken from the tables in Claret (2000). The use of Eq. (1) with the $K$ band coefficient provides a conversion factor $\rho$ of 1.027 which, in turn, yields a LD diameter for AB Dor A of $0.60 \pm 0.04$ mas. Given the small magnitude of this LD correction (less than 3\%), the uncertainties assigned to the coefficient $u(\lambda)$ (up to $10 \%$ ) do not significantly alter the radius estimate. Similarly, the use 


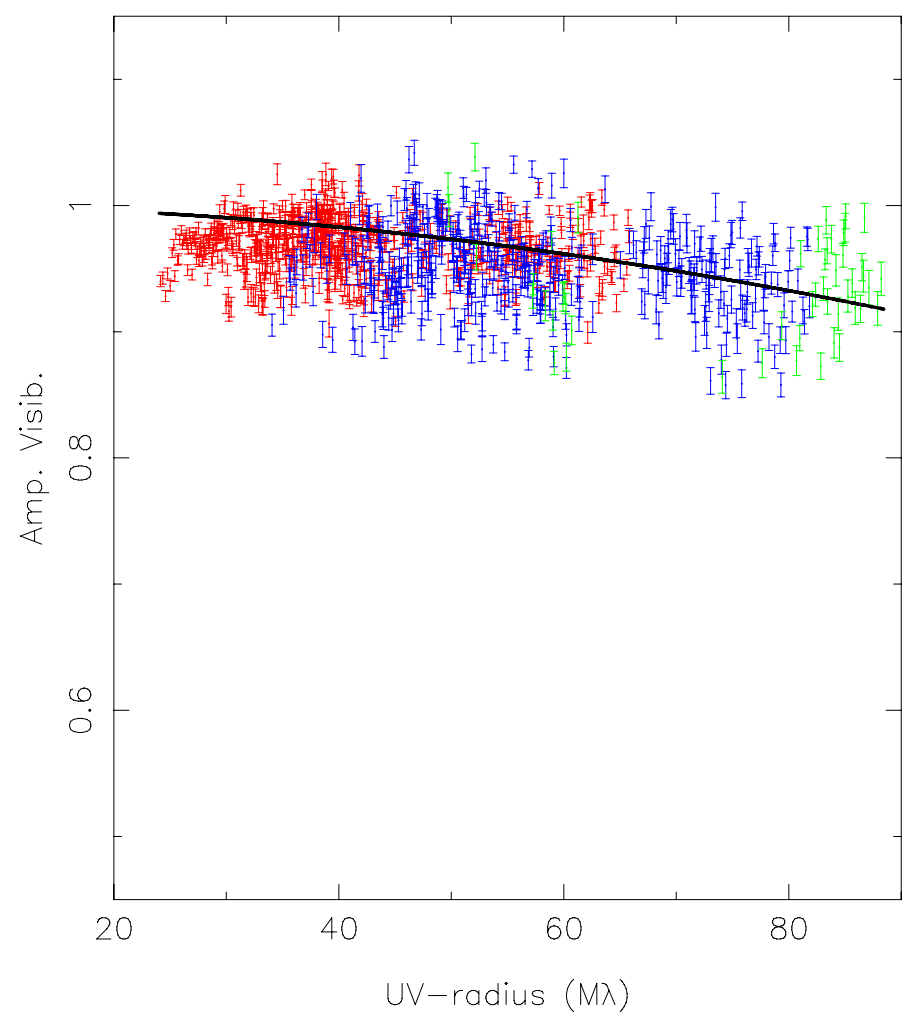

Fig. 1. AMBER/VLTI squared visibility amplitudes of AB Dor A for $J$, $H$, and $K$ bands (green, blue, and red data, respectively). The black continuous line corresponds to the visibilities resulting from the best fit of the data to a uniform disk model. The source is only partially resolved by the triplet A0-K0-G1, but sufficiently to constrain the angular size of $\mathrm{AB}$ Dor A to $0.62 \pm 0.04$ mas.

Table 2. Fundamental parameters of the PMS star AB Dor A.

\begin{tabular}{lc}
\hline \hline$K$ magnitude: & $4.686 \pm 0.016^{a}$ \\
Luminosity $\left(L_{\odot}\right):$ & $0.388 \pm 0.008^{a}$ \\
$T_{\text {eff }}(\mathrm{K})$ & $5081 \pm 50^{a}$ \\
$v \sin i\left(\mathrm{~km} \mathrm{~s}^{-1}\right)$ & $91 \pm 1^{b}$ \\
Distance $(\mathrm{pc}):$ & $14.9 \pm 0.1^{c}$ \\
Mass $\left(M_{\odot}\right):$ & $0.86 \pm 0.05^{d}$ \\
$\theta_{\mathrm{UD}}(\operatorname{mas}):$ & $0.62 \pm 0.04^{e}$ \\
$\theta_{\mathrm{LD}}(\operatorname{mas}):$ & $0.64 \pm 0.04^{e}$ \\
Radius $\left(R_{\odot}\right):$ & $0.96 \pm 0.06^{e}$ \\
\hline
\end{tabular}

References. ${ }^{(a)}$ Close et al. (2007). ${ }^{(b)}$ Collier-Cameron et al. (2002a). ${ }^{(c)}$ Guirado et al. (1997). ${ }^{(d)}$ Guirado et al. (2006). ${ }^{(e)}$ This paper.

of the $H$-band coefficients in Eq. (1) does not affect the LDdiameter by more of $0.3 \%$. Finally, using the distance measurement to this system, $14.9 \pm 0.1 \mathrm{pc}$ (Guirado et al. 2006), the LD angular diameter can be readily converted into a linear diameter of AB Dor A: $0.96 \pm 0.06 R_{\odot}$ (see Table 2 ). Given the extraordinary precision of the distance determination, we note that the uncertainty in the latter angular-to-linear conversion is just that corresponding to error propagation.

\subsection{Sub-structures in the visibility amplitude}

Despite the scatter of the measurements, the visibility amplitudes in Fig. 1 show small sinusoidal trends, up to $10 \%$, for $K$ and $H$ bands. Clearly, these substructures are not accounted for by our uniform disk model. A natural explanation could be the

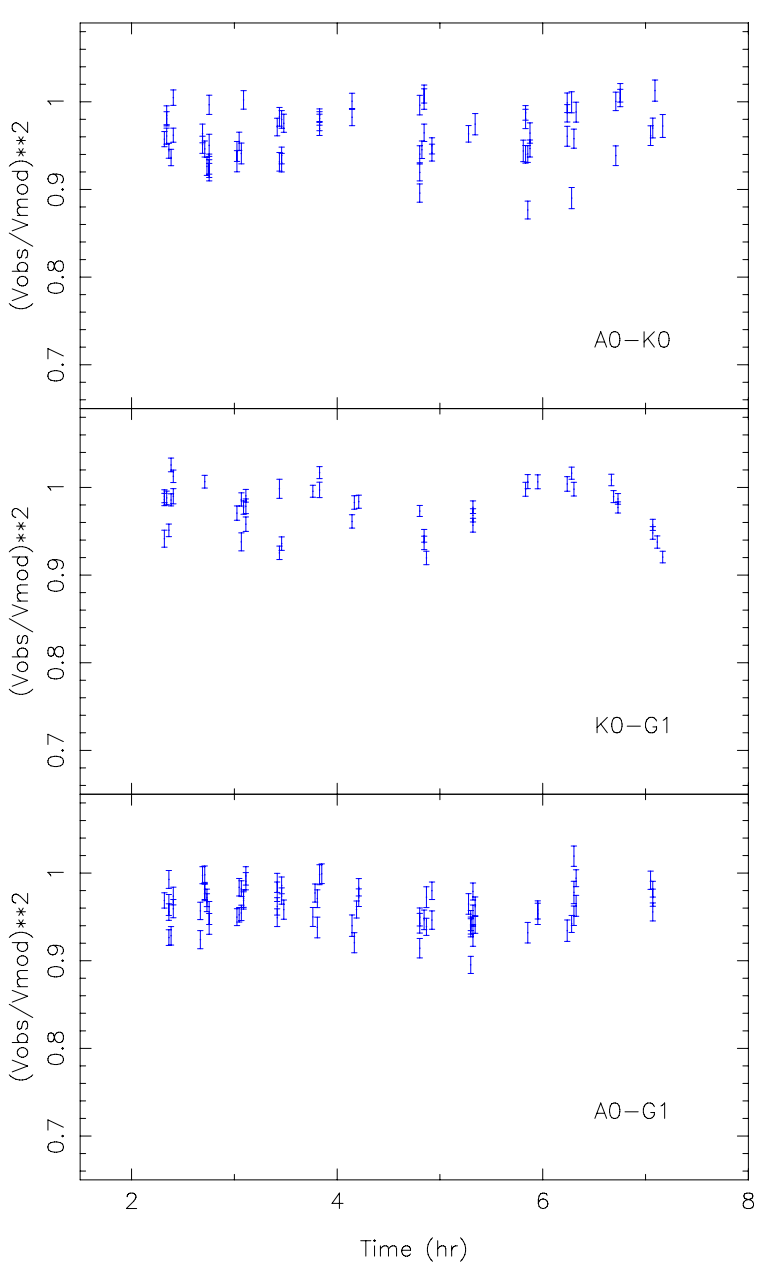

Fig. 2. Time evolution of the ratio between observed and modeled amplitude visibilities for the triplet A0-K0-G1 ( $H$-band). Data have been averaged in $0.5 \mathrm{M} \lambda$ bins in the $u v$-plane. These residuals uncover the substructures not accounted for by the uniform disc model. See text.

presence of the low-mass companion AB Dor C. However, this is not likely: the separation between $\mathrm{AB}$ Dor $\mathrm{A}$ and $\mathrm{AB}$ Dor $\mathrm{C}$ at the time of the VLTI observations is $\sim 300$ mas (calculated with the orbital parameters given in Guirado et al. 2006), which places $\mathrm{AB}$ Dor $\mathrm{C}$ out of the field-of-view of the AMBER instrument when used with the ATs ( 250 mas). Therefore, amplitude variations due to the small companion are not expected for such a particular position in the orbit to within the AMBER capabilities.

Rather, we consider that such substructures may be due to stellar activity on the surface on AB Dor A, most probably a combination of starspots and stellar rotation. The rotation and magnetic activity of the AB Dor A surface has been well studied (i.e. Collier-Cameron \& Donati 2002b; Cohen et al. 2010 and references therein), and the presence and evolution of starspots characterized. Typically, spots permanently cover a significant portion of the stellar surface; to complicate the scenario, the spots move quickly over the surface due to the fast rotation of AB Dor A. Since our observation time span is comparable to the rotation period of AB Dor A (0.514 days; Innis et al. 1998) it is not a surprise that the visibilities could be affected by such fast structural variations. Actually, this effect is better seen in Fig. 2, which shows the time evolution of the residuals of the amplitude visibilities, expressed as the ratio between the observed 
and modeled values, for the triplet A0-K0-G1 (for clarity, data have been averaged in $0.5 \mathrm{M} \lambda$ bins in the $u v$-plane). These residuals uncover the sinusoidal signature present in the amplitude visibilities that we assigned to the presence of starspots in fast rotation. We have carried out simple interferometric tests to verify the above statement: the starspots can be simulated by the addition of smaller disks on the surface of our uniform disk; such a smaller disks introduce an asymmetry in the AB Dor A structure, which, as seen by an interferometer, effectively produce a sinusoidal signature in the visibility amplitudes. However, more precise observations and a more elaborated model of the rotation, size, variability, and number of starspots in AB Dor A, both beyond the scope of this paper, appear necessary to properly clarify the origin of the subtle variations in our interferometric observables.

\section{Discussion}

\subsection{Comparison with PMS stellar models}

Our radius measurement of AB Dor A is in reasonable agreement with previous estimates based on other techniques, i.e. techniques based on the projected equatorial velocity $\left(\sim 1 R_{\odot}\right.$; Maggio et al. 2000), and techniques based on the empirical Barnes et al. (1978) relationship between radius and $V-R$ colors $\left(0.98 \pm 0.04 R_{\odot}\right.$; Collier-Cameron \& Foing 1997; Maggio et al. 2000). All these results indicate, as already pointed out by Collier-Cameron \& Foing (1997), that AB Dor A has not yet reached the main sequence, as its radius is larger than that for a ZAMS star of the same spectral type. AB Dor A is one of the few PMS stars having a very precise determination of many of its fundamental parameters (see Table 2); hence, they can be compared with the values resulting from PMS evolutionary models, both in the luminosity/effective temperature plane (H-R diagram) and in the mass/radius plane (M-R plane). Eventually, we should derive an age for the system both from the H-R diagram and for the M-R plane. We have used the PMS models from Barrafe et al. (1998; BCAH98) and Montalbán et al. (2004; MDKH04). The BCAH98 models use the NextGen atmospheres (Hauschildt et al. 1999) with a treatment of the convection based on the mixing length theory (MLT; Böhm-Vitense 1958). MLT convection is characterized by the mixing parameter $\alpha$, defined as $l_{\text {mix }} / H_{\mathrm{p}}$, with $l_{\text {mix }}$ the convective mixing length and $H_{\mathrm{p}}$ the pressure scale height; in particular, we used models with $\alpha=1$ and $\alpha=1.9$, the latter being required to match the solar values. In contrast, the MDKH04 models use the atmospheres from Heiter et al. (2002) with a convection treatment based on full spectrum turbulence (FST; Canuto \& Mazzitelli 1991; Canuto et al. 1996). We used FST models made to fit the solar values; this makes MDKH04 models more comparable to BCAH98 with $\alpha=1.9$.

In Fig. 3 we show the H-R diagram for the three models considered, where the placement of AB Dor A can be compared with theoretical isochrones and isomasses. As seen in the figure, BCAH98 models for $\alpha=1$ fail to predict any of the measurements of AB Dor A. However, both BCAH98 with $\alpha=1.9$ and MDKH04 models offer good predictions for both age and mass. Regarding the mass, both models predict a mass for AB Dor A below the dynamical mass estimate $\left(0.86 \pm 0.05 M_{\odot}\right)$, but well within the quoted uncertainties. The slight underprediction displayed in these two models is in agreement with that reported in Hillenbrand \& White (2004) and Mathieu et al. (2007), who pointed out that PMS models may underpredict masses below $1.2 M_{\odot}$. On the other hand, isochrones in H-R diagrams of
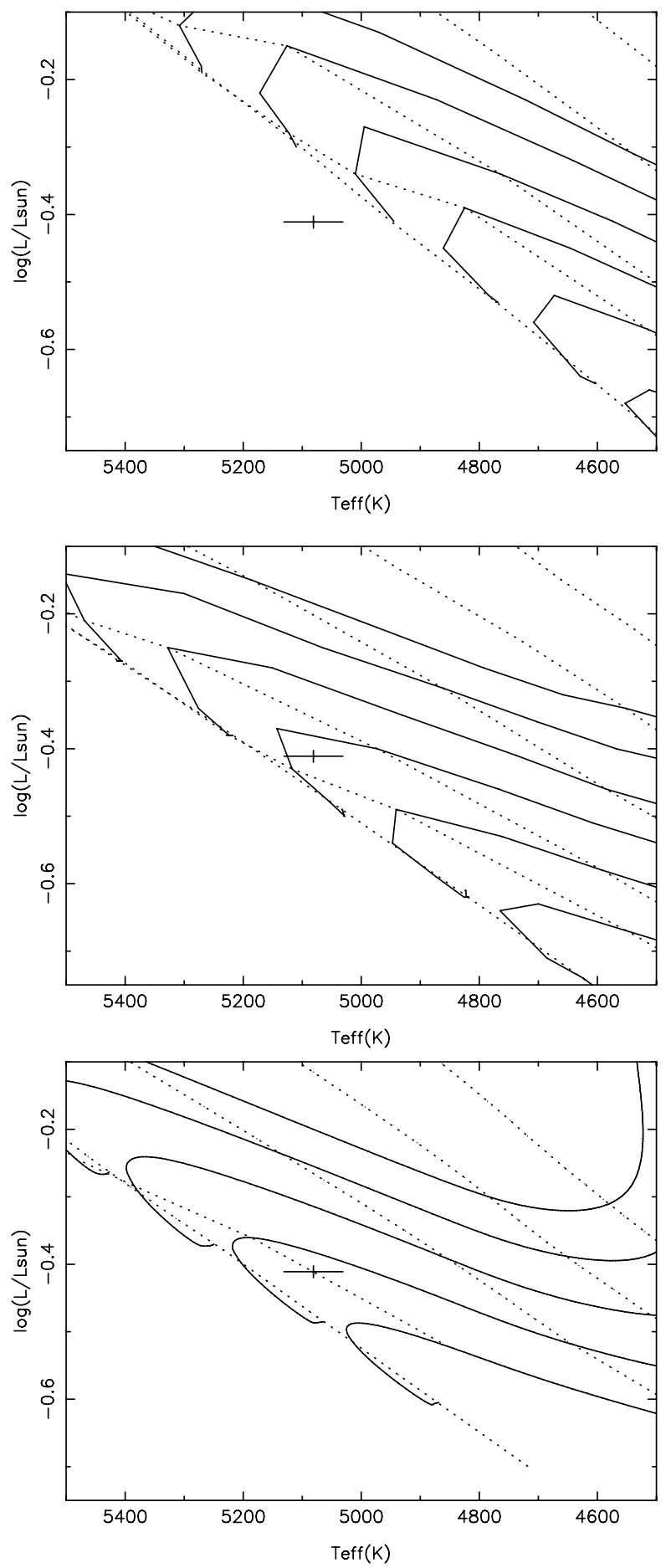

Fig. 3. H-R diagrams for several PMS evolutionary models. Isochrones (dotted lines) correspond to 10 (top isochrone), 16, 25, 40, and $100 \mathrm{Myr}$ (an additional $50 \mathrm{Myr}$ isochrone is shown in the middle plot). Isomasses (continuous lines) are for 0.75 (bottom isomass), 0.80, 0.85, 0.90, 0.95, and $1.0 M_{\odot}$. Points with error bars correspond to measurements (see Table 2). (Top) BCAH98 models, $\alpha=1$. (Middle) BCAH98 models, $\alpha=1.9$ (solar value). (Bottom) MDKH04 models.

BCAH98 with $\alpha=1.9$ and MDKH04 seem to favor an early age for AB Dor A (a range of 40-50 Myr covers the estimates of both models). However, as we will see below, success (or failure) in the H-R diagram may not translate to the M-R plane.

Comparisons in the M-R plane could be considered even more fundamental than those in the H-R diagram, as they depend 

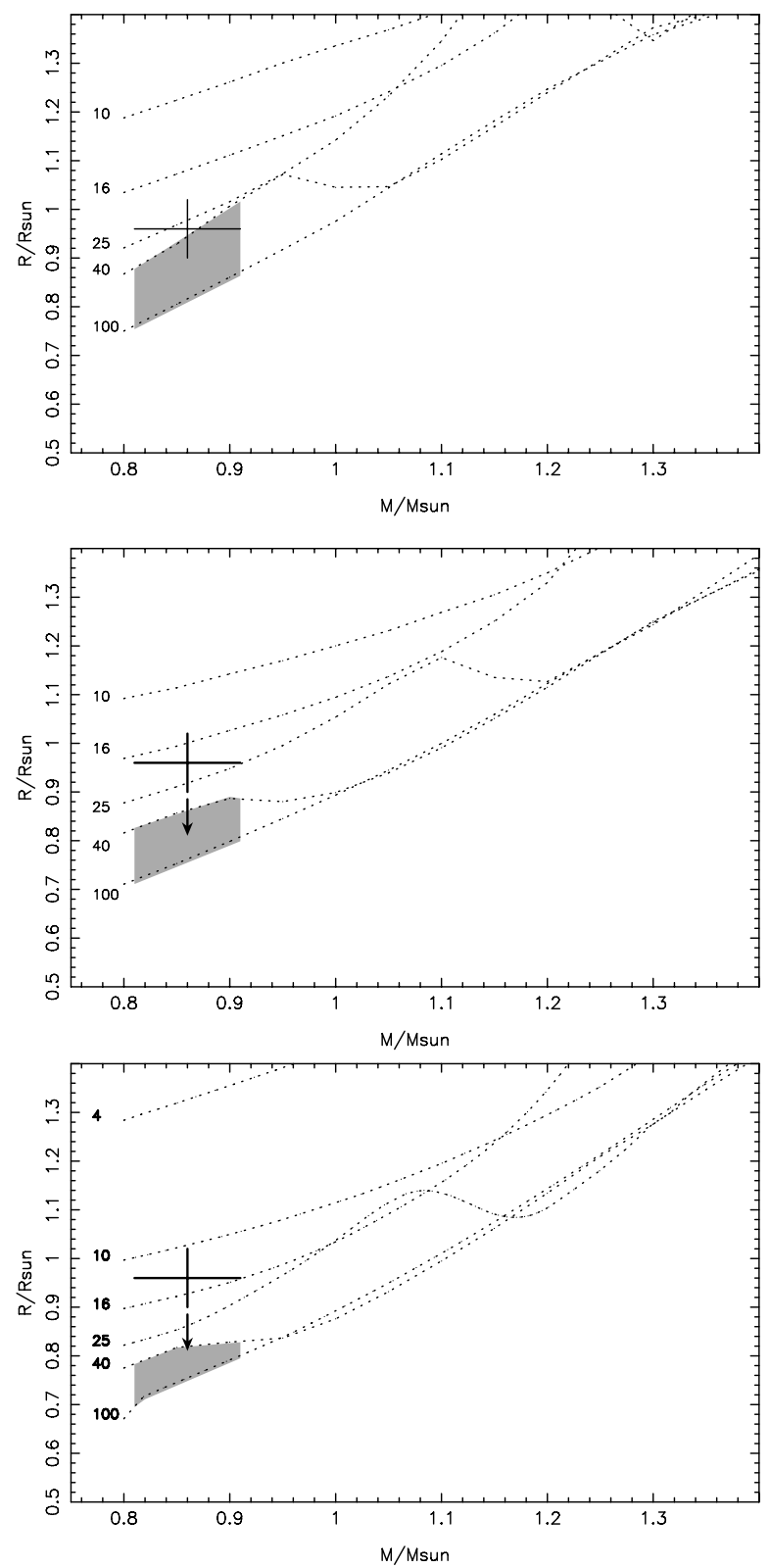

Fig. 4. Mass-Radius relationship for several PMS evolutionary models. Isochrones (in Myr) are shown as dotted lines. Points with error bars correspond to measurements. The shadowed area indicates the age range considered for the low-mass companion AB Dor C (40-120 Myr). Arrows in middle and bottom plots indicate the "magnetic free" radius (see text). (Top) BCAH98 models, $\alpha=1$. (Middle) BCAH98 models, $\alpha=1.9$ (solar value). (Bottom) MDKH04 models.

only of the accuracy of the measurements, free from ambiguities related to the determination of effective temperatures (Mathieu et al. 2007). In Fig. 4, our measurement of both mass and radius for $\mathrm{AB}$ Dor A are shown along with isochrones corresponding to the same models as in Fig. 3. The shadowed area corresponds to a generous range of possible ages for $\mathrm{AB}$ Dor $\mathrm{C}$, the lowmass companion of AB Dor A (40-120 Myr; see Sect. 1). In contrast to the results obtained above in the H-R diagram, BCAH98 models with $\alpha=1$ seem to agree, within uncertainties, with the younger side of the AB Dor C age interval, while BCAH98 with $\alpha=1.9$ and MDKH04 models predict an age for AB Dor A which is perhaps too young (16-25 Myr and 10-16 Myr, respectively) if we posit that $\mathrm{AB}$ Dor $\mathrm{A}$ and $\mathrm{AB}$ Dor $\mathrm{C}$ are coeval. This apparent mismatch between the predictions of the H-R diagram and the M-R plane has been already reported for other stars (i.e. Stassun et al. 2004). In the case of AB Dor A, the strong magnetic activity of this star may play an important role in the predictions of both the H-R diagram and the M-R plane. We discuss this in turn.

\subsection{The role of the magnetic field}

There is some evidence that the magnetic activity may influence the evolution and structure of PMS objects. Torres et al. (2006) found some disagreement $(\sim 10-15 \%)$ between predicted and modeled radius for PMS active eclipsing binaries which was not for non-active stars. The connection between magnetic activity and size has been reported earlier (Ribas 2003; Torres et al. 2006 and references therein): in essence, the stellar convective heat transport is inhibited by the presence of a strong magnetic field, usually related to a rapid stellar rotation, and by the frequency and duration of spots in the stellar surface. As a consequence of this loss of efficiency of the convection, the star must augment its size to radiate the accumulated energy; therefore, magnetically active stars would have larger radii than those estimated in absence of magnetic field. Following the same line of reasoning, in the framework of the mixing length treatment of convection, a less efficient convection corresponds to a lower value of the mixing parameter $\alpha$ (Tayler 1987). In fact, Torres et al. (2006) also found that models with lower $\alpha$ agree better with the estimated size of active stars.

AB Dor A is a highly-magnetized (surface values of $200 \mathrm{G}$ on average; Cohen et al. 2010), fast-rotating star and certainly a good candidate to suffer the effects of a strong magnetic field on its evolution. Actually, the BCAH98 isochrones for $\alpha=1$ are compatible at the same time with the measured radius of $\mathrm{AB}$ Dor $\mathrm{A}$ and, to within uncertainties, with the age interval of $\mathrm{AB}$ Dor $\mathrm{C}$ (see Fig. 4). On the other hand, the radius of AB Dor A predicted by the BCAH98 with $\alpha=1.9$ and MDKH04 in the age interval of $\mathrm{AB}$ Dor $\mathrm{C}$ is smaller than that measured. Hence, assuming coevality, the available data indicate that convection in AB Dor A must be less efficient than in the Sun, and that the required value of the mixing length parameter to fit the radius of $\mathrm{AB}$ Dor $\mathrm{A}$ is smaller than that required to fit the radius of the Sun at its present age. Based on the results of Torres et al. (2006), we can estimate a "magnetic-free" radius of AB Dor A allowing for an oversizing factor of $15 \%$ in our interferometric measurements as a consequence of the strong magnetic activity. If we translate this "magnetic-free" radius $\left(\sim 0.81 R_{\odot}\right)$ to the M-R plane representations (indicated by arrows in Fig. 4, middle and bottom plots), the measurements would be placed well in the range of the $\mathrm{AB}$ Dor $\mathrm{C}$ age, actually in agreement with the age predicted by the same models from the H-R diagrams (40-50 Myr).

This agreement between the age estimates from H-R diagrams and $\mathrm{M}-\mathrm{R}$ planes seems to favour the younger side of the $\mathrm{AB}$ Dor $\mathrm{C}$ range as the most probable age for the $\mathrm{AB}$ Doradus system, substantially younger than that of the Pleiades cluster ( 120 Myr; Luhman et al. 2005). Our result is marginally compatible with the latter, older age, just at the extreme end of the uncertainties associated with our radius measurement.

It should be mentioned that the fast rotation of $\mathrm{AB}$ Dor $\mathrm{A}$ has further consequences beyond those related with the magnetic field. The most direct effect is that the high rotation may alter the oblateness of the star producing a larger radius at the equator than at the poles. In turn, the oblateness the star leads to gravitational darkening (von Zeipel 1924); according to this effect, both the surface gravity and the brightness of the star 


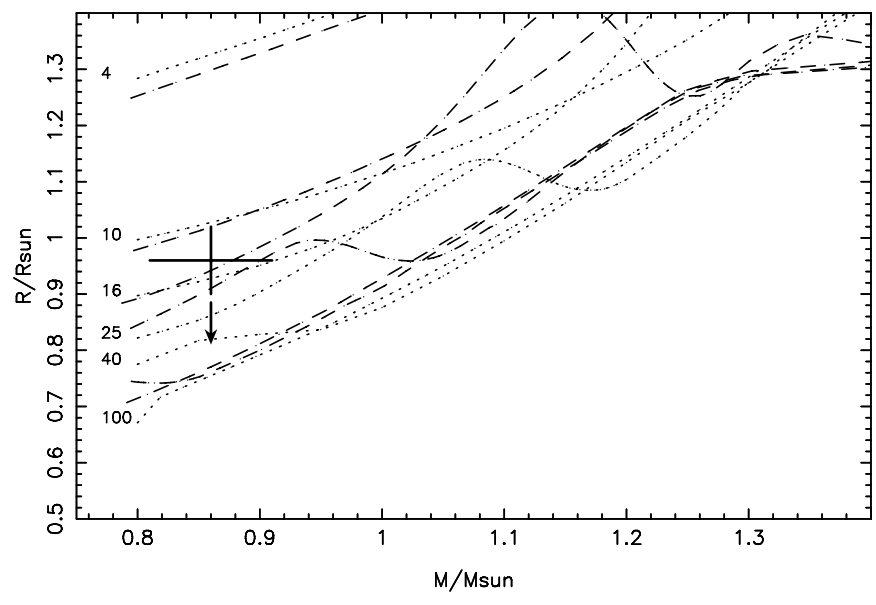

Fig. 5. Mass-Radius relationship for different metallicities (MDKH04 models). Isochrones (in Myr) are shown for $[\mathrm{M} / \mathrm{H}]=0$ (dotted lines) and $[\mathrm{M} / \mathrm{H}]=-0.3$ (dashed line, which corresponds to the AB Dor A metallicity reported in Gómez de Castro 2002).

decrease from the poles to the equator. Both effects could be present in $\mathrm{AB}$ Dor $\mathrm{A}$ since, in fact, there are evidences of a time-dependent oblateness in this object (Collier-Cameron \& Donati 2002b). On the other hand, oblateness and gravitational darkening have been measured from IR interferometry for other stars like Achernar or Altair (Domiciano de Souza et al. 2003, 2005). However, the above-mentioned stars subtend an angular size $\sim 5$ times larger than that of $\mathrm{AB}$ Dor A ( $0.62 \pm 0.04$ mas), which is only partially resolved by our AMBER/VLTI observations (see Fig. 1). Therefore, the detection of these fine details of the structure of $\mathrm{AB}$ Dor $\mathrm{A}$ would require a longer and more sensitive interferometer that should shed some light on these important, rotationally-related, contributions.

Finally, our angular size estimate of AB Dor A can be combined with the bolometric flux to obtain a direct measurement of the effective temperature from the Stephan-Boltzmann law. For this purpose we have used a bolometric correction at $K$-band, $\mathrm{BC}(K)=1.9 \pm 0.1$, obtained from the polynomial fits of Masana et al. (2008). The resulting value of the effective temperature is $4800 \pm 300 \mathrm{~K}$, coincident, within uncertainties, to the temperature reported in Table 2. We note that the large uncertainties of this effective temperature mostly correspond to the $\sim 6 \%$ error in the radius estimate.

\subsection{Influence of the metallicity}

Gómez de Castro (2002) reported a metallicity for AB Dor A of $[\mathrm{M} / \mathrm{H}]=-0.3$. We have used the MDKH04 models to evaluate the effect of the metallicity in the M-R plane. In Fig. 5 we show the isochrones for both solar composition and $[\mathrm{M} / \mathrm{H}]=-0.3$. The age inferred from our measurement is not altered significantly following one or other value of the metallicity. As done in Fig. 4, an arrow accounts for the $15 \%$ oversizing, which points to ages younger than $40 \mathrm{Myr}$. However, we notice that, for $[\mathrm{M} / \mathrm{H}]$ $=-0.3$, isochrones are packed between 40 and $100 \mathrm{Myr}$, making it difficult to choose a particular age within that range.

\section{Conclusions}

We present new AMBER/VLTI interferometry observations determining the size of the PMS star AB Dor A. We have used a simple model (uniform disk) to fit the interferometric visibilities and derive an angular diameter of $0.62 \pm 0.04$ mas. The corresponding limb-darkened value $(0.64 \pm 0.04$ mas $)$, combined with a very precise previous estimate $(14.9 \pm 0.01 \mathrm{pc})$, allows for a most precise measurement of the linear radius $\left(0.96 \pm 0.06 R_{\odot}\right)$. Some (weak) substructures are also apparent in the visibilities (sinusoidal variations up to $10 \%$ in amplitude). We consider that these sinusoidal variations might be produced by stellar spots, which are frequent and intense in $\mathrm{AB}$ Dor $\mathrm{A}$ as a consequence of its chromospheric activity. The fast rotation rate of AB Dor A, comparable to our observation time span, may play a role too, masking the visibility trends. The combination of dynamical mass and this new radius determination facilitates the comparison of these two fundamental parameters with those provided by theoretical PMS stellar models. We have found evidence of disagreement between the predictions based on H-R diagrams and those based on M-R planes. Part of this discrepancy could be due to the strong magnetic field on the surface of AB Dor A, which, as other authors point out and our results show, may inhibit the efficiency of the convection and produce a larger radius than predicted by PMS models calibrated to fit the radius of the Sun. Should this magnetic effect be accounted for, we could reconcile the predictions from the H-R diagram and the M-R plane for the models considered in this paper (except the H-R predictions of BCAH98 models with $\alpha=1$ ), favouring an age for AB Dor A of 40-50 Myr, at the younger end of the range of published ages of the low-mass companion AB Dor C. Older ages are not completely excluded by our work, although coevality with the Pleiades cluster appears to be marginal and only compatible with our data at the extreme end of the (somewhat conservative) size uncertainties. Finally, we notice that, with this new estimate of the linear radius, AB Dor A is one of the few PMS stars with most of the fundamental parameters precisely determined (see Table 2). This makes AB Dor A a very appropriate object to check the consistency of PMS models, and in particular, those intended to include the effects of the magnetic activity in stellar evolution.

Acknowledgements. This work has been partially founded by grant AYA200913036-C02-02 of the Spanish MICINN, and by grant PROMETEO 104/2009 of the Generalitat Valenciana. The National Radio Astronomy Observatory is operated by Associated Universities, Inc. under cooperative agreement with the (US) National Science Foundation. This research has made use of the SIMBAD database, operated at CDS, Strasbourg, France.

\section{References}

Barnes, T. G., Evans, D. S., \& Moffett, T. J. 1978, MNRAS, 182, 285

Barrafe, I., Chabrier, G., Allard, F., \& Hauschildt, P. H. 1998, A\&A, 337, 403 (BCAH98)

Boccaletti, A., Chauvin, G., Baudoz, P., \& Beuzit, J.-L. 2008, A\&A, 482, 939

Böhm-Vitense, E. 1958, Z. Astrophys., 46, 108

Canuto, V. M., \& Mazzitelli, I. 1991, ApJ, 370, 295

Canuto, V. M., Goldman, I., \& Mazzitelli, I. 1996, ApJ, 473, 550

Claret, A. 2000, A\&A, 363, 1081

Close, L. M., Lenzen, R., Guirado, J. C., et al. 2005, Nature, 433, 286

Close, L. M., Niranjan, T., Nielsen, E. L., et al. 2007, ApJ, 665, 736

Cohen, O., Drake, J. J., Kashyap, G. A., et al. 2010, ApJ, 721, 80

Collier-Cameron, A., \& Donati, J.-F. 2002b, MNRAS, 329, L23

Collier-Cameron, A., \& Foing, B. H. 1997, Observatory, 117, 218

Collier-Cameron, A. Donati, J.-F., \& Semel, M. 2002a, MNRAS, 330, 699

Di Folco, E., Thévenin, F., Kervella, P., et al. 2004, A\&A, 426, 601

Domiciano de Souza, A., Kervella, O., Jankov, S., et al. 2003, A\&A, 407, L47

Domiciano de Souza, A., Kervella, O., Jankov, S., et al. 2005, A\&A, 442, 567

Domiciano de Souza, A., Bendjoya, P., Vakili, F., et al. 2008, A\&A, 489, L5 
J. C. Guirado et al.: The size of AB Doradus A from VLTI/AMBER interferometry

Gómez de Castro, A. I. 2002, MNRAS, 332, 409

Guirado, J. C., Reynolds, J. E., Lestrade, J.-F., et al. 1997, ApJ, 490, 835 Guirado, J. C., Martí-Vidal, I., \& Marcaide, J. M. 2006, A\&A, 446, 733

Hanbury-Brown, R., Davis, J., \& Allen, L. R. 1974, MNRAS, 167, 121

Hauschildt, O. H., Allard, F., \& Baron, E. 1999, ApJ, 512, 377

Heiter, U., Kupka, F., van't Veer-Menneret, C., et al. 2002, A\&A, 392, 619

Hillenbrand, \& White 2004, ApJ, 604, 741

Innis, J. L., Thompson, K., Coates, D. W., \& Evans, T. L. 1998, MNRAS, 235, 1411

Janson, M., Brandner, W., Lenzen, R., et al. 2007, A\&A, 462, 615

Kraus, S., Weigelt, G., Balega, Y. Y., et al. 2009, A\&A, 497, 195

López-Santiago, J., Montes, D., Crespo-Chacón, I., \& Fernández-Figueroa, M. J. 2006, A\&A, 643, 1160

Luhman, K. L., Stauffer, J. R., \& Mamajek, E. 2005, ApJ, 628, L69

Maggio, A., Pallavicini, R., Reale, F., \& Tagliaferri 2000, A\&A, 356, 627

Masana, E., Jordi, C., \& Ribas, I. 2006, A\&A, 450, 735
Martí-Vidal, I., Marcaide, J. M., Quirrenbach, A., et al. 2011, A\&A, 529, A115 Mathieu, R. D., Baraffe, I., Simon, M., et al. 2007, Protostars and Planets V, 951, 411

Mérand, A., Bordé, P., \& Coudé du Foresto, V. 2006, A\&A, 447, 783

Montalbán, J., D’Antona, F., Kupka, F., \& Heiter, U. 2004, A\&A, 416, 1081 (MDKH04)

Nielsen, E., Close, L. M., Guirado, J. C., et al. 2005, AN, 326, 1033

Ortega, V., Jilinski, E., de La Reza, R., \& Bazzanella, B. 2007, MNRAS, 377, 441

Petrov, R. G., Malbet, F., Weigelt, G., et al. 2007, A\&A, 464, 1 Stassun, K. G., Mathieu, R. D., Vaz, L. P. R., et al. 2004, ApJ, 151, 357

Tatulli, E., Millour, F., Chelli, A., et al. 2007, A\&A, 464, 29

Tayler, R. J. 1987, MNRAS, 227, 553

Torres, G., Lacy, C. H., Laurence, A., et al. 2006, ApJ, 640, 1018

von Zeipel, H. 1924, MNRAS, 84, 665

Zuckerman \& Song 2004, ARA\&A, 42, 685 